TRABAJOS ORIGINALES

\title{
Patrones de coocurrencia y conducta alimentaria a escala local de Phlebotominae (Diptera: Psychodidae) del estado Falcón, Venezuela
}

\section{Co-ocurrence patterns and feeding behaviour at local scale of Phlebotominae(Diptera: Psychodidae) from Falcon state, Venezuela}

\author{
Dalmiro J. Cazorla*, Elsa Nieves ${ }^{2}$ y Pedro Morales ${ }^{1}$
}

\section{${ }^{*}$ Autor para correspondencia:}

1 Laboratorio de Entomología, Parasitología y Medicina Tropical, Centro de Investigaciones Biomédicas, Universidad Nacional Experimental "Francisco de Miranda" (UNEFM), Apdo. 7403, Coro 4101, Estado Falcón, Venezuela.

2 Laboratorio de Parasitología Experimental, Facultad de Ciencias, Departamento de Biología, Universidad de Los Andes, Mérida, Estado Mérida, Venezuela

Email Dalmiro J. Cazorla: lutzomyia@hotmail.com Email Elsa Nieves: nievesbelsa@gmail.com

Email Pedro Morales:

pemoralesmoreno@hotmail.com

Citación:

Cazorla D.J., E. Nieves \& P. Morales. 2014. Patrones de coocurrencia y conducta alimentaria a escala local de Phlebotominae (Diptera: Psychodidae) del estado Falcón, Venezuela. Rev. peru. biol. 21(1): 099-104 (Mayo 2014). doi: http://doi. org/10.15381/rpb.v21i1.8253

\section{Resumen}

Los flebotominos son transmisores de los protozoarios parásitos del genero Leishmania, agentes causales de las leishmaniasis en humanos y otros mamíferos. Mediante modelos nulos, se estudio la estructura de las comunidades flebotominas en focos endémicos de leishmaniasis del estado Falcón, en el nor-occidente de Venezuela, a una escala reducida o local: en el domicilio, peridomicilio y el área silvestre de una zona de vida o en una localidad en particular. La aplicación de los modelos nulos reveló que a escala local las comunidades flebotominas se encuentran agregadas, sugiriendo que las especies coexisten y no compiten. Los estudios de co-ocurrencia con el análisis de estructura gremial y la prueba de la hipótesis de los estados favorecido mostró que los resultados obtenidos no son estadísticamente significativos ( $p>0.05$ ), lo que sugiere que las especies flebotominas pertenecen a un mismo gremio en sus preferencias alimentarias, lo que podría deberse a que la hematofagia se trata de un evento heterogéneo, circunstancial y oportunista. Se discuten aspectos sobre los posibles factores, como por ejemplo la transformación y homogenización de los hábitats por el impacto sinantrópico, que pudieran estar determinando el ensamble de los flebotominos en la región falconiana.

Palabras clave: Flebotominos; modelos nulos; Leishmaniasis; Venezuela.

\section{Abstract}

The phlebotomine sandfly are vectors of protozoan parasites Leishmania genus, the causative agents of leishmaniasis in humans and several mammalian hosts. The structure of the phlebotomine sandfly community at the local scale: domicile, peridomicile and sylvatic habitats from a reduced area, was assessed using null models analysis in endemic foci of leishmaniasis from Falcon state, in north-western region of Venezuela. Implementation of null model tests revealed that to a local scale the phlebotomine sandfly communities are aggregated, suggesting that the species co-occurred and did not compete. The assays of co-ocurrence patterns with guild structure analysis and favored states hypothesis showed statistically nonsignificant results ( $p>0.05$ ), suggesting that sandfly species belong to a same guild in relation to their feeding preferences, as hematophagy appears to be an heterogeneous, circumstantial and opportunist event. We discussed aspects of the possible factors, for example sinantropic effects on environmental transformation and its homogenization, that could be determining the phlebotomine sanfly assemblage in the Falcon state region.

Keywords: Sandflies; Null models; Leishmaniasis; Venezuela. 


\section{Introducción}

Las leishmaniasis son entidades nosológicas producidas por varias especies de protozoarios flagelados del género Leishmania sp., y que se transmiten a varios hospedadores vertebrados (Mammalia) y al humano por la picadura de insectos dípteros (Psychodidae) de la subfamilia Phlebotominae (Young \& Duncan 1994, Herrera 2010). Estas dolencias se encuentran presentes prácticamente en toda la geografía venezolana, realidad a la que no escapa el estado Falcón (región nor-occidental), al cual se le considera un foco activo tanto de leishmaniasis tegumentaria (LT) como de su forma visceral (LV). En el estado Falcón se han detectado tasas de incidencia de LT de 2.09 por cada 100000 habitantes (De Lima et al. 2010), y varios casos activos de kalaazar, especialmente en infantes, patología que si no es tratada a tiempo es potencialmente mortal (Añez et al. 2012).

En los focos endémicos de LT y LV del estado Falcón se ha registrado una co-ocurrencia de hasta 24 especies del género Lutzomyia França 1924, algunas de las cuales poseen conductas alimentarias consideradas como antropofilícas y otras como zoofílicas (Young \& Duncan 1994, Añez et al. 2012, Cazorla \& Morales 2012). Se considera que el balance entre estas dos conductas alimentarias debe jugar un papel importante en la dinámica de transmisión de las leishmaniasis, al ser las especies flebotominas zoofílicas las que introducen a Leishmania en los ambientes sinantrópicos, y luego las especies antropofílicas esparcen la infección a los humanos (Chaves \& Añez 2004).

Uno de los tópicos que ecología de comunidades presta a más atención es la identificación de los mecanismos generales que gobiernan la estructura de las comunidades biológicas ("reglas de ensamble”), y aunque se han propuesto varias reglas se le ha dado mayor relevancia a las interacciones interespecíficas (competencia, mutualismo) (Vilchis 2000, Chase 2003, Feeley 2003). Sin embargo, es necesario indicar que la coexistencia entre las especies puede estar gobernada por fuerzas competitivas y no competitivas (Gordon 2000).

Los modelos nulos son métodos estadísticos y simulaciones computarizadas que se usan para indagar o analizar si existe el azar en los procesos de la naturaleza que generan algún patrón ecológico, de aquí que se han implementado para estudiar los patrones de coocurrencia (Gotelli 2000, Gotelli \& Entsminger 2003). Los modelos nulos trabajan con datos ecológicos aleatorizados procedentes de un modelo conocido o imaginario, de manera tal que se puede determinar si los patrones observados en las comunidades biológicas son gobernados por eventos o sucesos ecológicos o evolutivos, o simplemente por el azar (Gotelli 2000, Gotelli \& Entsminger 2003).

El análisis de las fuentes bibliográficas relacionadas con los estudios ecológicos sobre la dinámica de transmisión de las leishmaniasis en Venezuela, revela que tan sólo se ha realizado un trabajo que involucra la estructura del ensamble de las comunidades flebotominas, el cual fue realizado en la región andina a diferentes pisos altitudinales y zonas bioclimáticas (i.e., a gran escala o regional) (Chaves \& Añez 2004). Por lo tanto, existe un desconocimiento de los patrones de coocurrencia de las comunidades flebotominas a escala local en el territorio de Venezuela, y particularmente en el estado Falcón. En este sentido, a sabiendas de que el bosque primario tropical es un factor relevante para la presencia de Lutzomyia (González-Salazar et al. 2013), es importante conocer el efecto que pudiera tener la alteración de estas áreas silvestres (e.g, construcción de asentamientos humanos) sobre la estructura de las comunidades flebotominas, tal como se ha documentado en focos endémicos de leishmaniasis del Paleotrópico (Italia) y Neotrópico (Panamá) (Chaves 2011). Este tipo de información puede aportar conocimiento útil para diseñar eficientemente un plan para el control vectorial de estas parasitosis (Chaves 2011).

A la luz de lo expuesto, en el presente trabajo mediante modelos nulos se determinó la estructura de las comunidades de especies flebotominas en focos endémicos de LT y/o LV del estado Falcón, Venezuela, a una escala reducida, i.e. en el domicilio, peridomicilio y el área silvestre de una zona de vida o en una localidad en particular (escala local).

\section{Material y métodos}

Área de estudio y datos.- Para estimar la estructura de comunidades flebotominas a escala local se tomaron los datos de un trabajo previo (Cazorla \& Morales 2012), el cual se realizó en 41 localidades ubicadas a diferentes pisos altitudinales y zonas bioclimáticas del estado Falcón $\left(10^{\circ} 18^{\prime} 08^{\prime \prime}\right.$ y $12^{\circ} 11^{\prime} 46^{\prime} \mathrm{N}$ y los $68^{\circ} 14^{\prime} 28^{\prime \prime}$ y $\left.71^{\circ} 18^{\prime} 21^{\prime \prime} \mathrm{W}\right)$, en la región noroccidental de Venezuela. Para ello, se escogieron las especies flebotominas capturadas en ambientes del domicilio, peridomicilio y silvestre de diez localidades incluyendo Guamacho $11^{\circ} 21^{\prime} 15^{\prime}$ "N y $\left.69^{\circ} 04^{\prime} 32^{\prime \prime} \mathrm{W}\right)$, Tocópero (1130'19,40"N y 69¹3'29,17”W), La Cienaguita

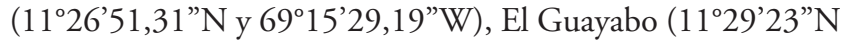
y $\left.69^{\circ} 15^{\prime} 33^{\prime \prime W}\right)$, Píritu $\left(11^{\circ} 21^{\prime} 58,13^{\prime \prime N}\right.$ y $\left.69^{\circ} 8^{\prime} 10,26^{\prime \prime W}\right)$, El Caballo $\left(11^{\circ} 29^{\prime} 9,79^{\prime} \mathrm{N}\right.$ y 69²10'50,90”W), Guaibacoa

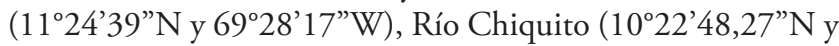
70³8'58,64”W), La Ciénaga (11²5’47,93”N y 69²7’34,20”W) y Chipare $\left(11^{\circ} 25^{\prime} 47,95^{\prime \prime} \mathrm{N}\right.$ y $\left.69^{\circ} 27^{\prime} 34,21^{\prime \prime W}\right)$, ubicadas entre 66 - $330 \mathrm{~m}$ de un área bioclimática en particular: bosque muy seco tropical (BMST); así mismo, se indagó los patrones de coocurrencia de las comunidades flebotominas en una localidad en particular: “San Francisco" (11 26'42,46”N y 69¹6’26,31”W), ubicada a $198 \mathrm{~m}$, similarmente en el BMST de la región falconiana (Ewel et al. 1976).

En las Tablas 1 y 2 se da el listado de las especie flebotominas estudiadas, así como datos acerca de su distribución por sitio de captura (domicilio, peridomicilio, área silvestre), preferencias alimentarias y métodos de captura (Young \& Duncan 1994, Cazorla \& Morales 2012). Tomándose como base la información de las Tablas 1 y 2 , para cada especie flebotomina se organizaron los datos empleando matrices presencia (1)-ausencia (0), las cuales son las unidades básicas fundamentales en los estudios biogeográficos y de ecología de comunidades (Gotelli 2000).

Modelos nulos.- Como índice de coocurrencia para determinar y cuantificar la estructura de las comunidades flebotominas, se utilizó el $C$-score o índice tablero de damas (checkerboard) de la matriz (Stone \& Roberts 1990, Gotelli 2000).

Se hicieron simulaciones computacionales tomándose los datos para todos los sitios de captura (domicilio, peridomicilio, área silvestre), con la totalidad de los métodos de captura (Cazorla \& Morales 2012). Para detectar si hubo sesgo debido a los métodos de captura empleados, similarmente se hicieron cálculos computacionales pero con datos particulares de los sitios de captura, para cada una de las técnicas de captura (Chaves \& Ańez 2004). Adicionalmente, se indagaron los patrones de coocurrencia en 
Tabla 1. Registro de preferencias alimentarias de especies flebotominas y su distribución por sitio de captura en 10 localidades endémicas de leishmaniasis del Bosque Muy Seco Tropical (BMST) del estado Falcón, Venezuela.

\begin{tabular}{|c|c|c|c|}
\hline Especie de Lutzomyia & Preferencia Alimentaria* & Sitio de captura** & Técnica de captura+ \\
\hline L. evansi & A & S, P, D & $\mathrm{TL} ; \mathrm{CH} ; \mathrm{AD}$ \\
\hline L. gomezi & A & $\mathrm{P}$ & $\mathrm{TL} ; \mathrm{CH} ; \mathrm{AD}$ \\
\hline L. longipalpis s.l. & A & $S, P, D$ & TL; PA \\
\hline L. lichyi & A & $\mathrm{S}$ & $\mathrm{AD}$ \\
\hline L. atroclavata & $\mathrm{Z}$ & S & $\mathrm{AD} ; \mathrm{PA}$ \\
\hline L.c. cayennensis & $\mathrm{Z}$ & $\mathrm{S}, \mathrm{P}$ & $\mathrm{TL} ; \mathrm{AD} ; \mathrm{PA}$ \\
\hline L. trinidadensis & Z & S, P & $\mathrm{TL} ; \mathrm{AD} ; \mathrm{PA}$ \\
\hline L. venezuelensis & Z & S & $\mathrm{AD} ; \mathrm{PA}$ \\
\hline
\end{tabular}

Tabla 2. Registro de preferencias alimentarias de especies flebotominas y su distribución por sitio de captura en "San Francisco", estado Falcón, Venezuela.

\begin{tabular}{lccc}
\hline \multicolumn{1}{c}{ Especie de Lutzomyia } & Preferencia Alimentaria* & Sitio de captura** & Técnica de captura+ \\
\hline L. evansi & A & S, P, D & TL; CH; AD \\
L. gomezi & A & P & TL; CH; AD \\
L. longipalpis s.l. & A & D,P & TL; PA \\
L. atroclavata & Z & S & AD; PA \\
L.c. cayennensis & Z & P,S & TL; AD; PA \\
L. trinidadensis & Z & P,S & TL; AD; PA \\
\hline
\end{tabular}

*Preferencias alimentarias: antropofílico (A); zoofílico (Z); datos basados en Young y Duncan (1994) y Cazorla y Morales (2012). +Técnica de captura: papel aceitado (PA); Aspiración Directa (AD); Trampa Lumínica (TL); Cebo Humano (CH). ** Sitios de captura: área selvática (S), peridomicilio (P) y domicilio (D); datos basados en Cazorla y Morales (2012).

las comunidades flebotominas para todos los sitios de captura con la totalidad de las técnicas, y para los sitios de captura y las técnicas de muestreo por separado, pero determinándose si existe sesgo con las preferencias alimentarias de las hembras flebotominas (Chaves \& Añez 2004). Para ello se realizó un análisis de estructura gremial (guild structure analysis), empleándose como índice para cuantificar los patrones de coocurrencia la variación del $C$-score (si la variación es baja, entonces los gremios poseen patrones de coocurrencia muy similares), siendo los gremios (guilds) las preferencias "antropofílicas" y las "zoofílicas" de las hembras flebotominas. Los gremios ecológicos son grupos de especies dentro de una comunidad que comparten recursos que les son comunes: es más probable que especies dentro de un mismo gremio interactúen o compitan por recursos que las de diferentes gremios (Gotelli 2000; Gotelli \& Entsminger 2003). Asimismo, se realizó la prueba de la hipótesis de los estados favorecidos (Fox 1987), y que no es más que una variante más estricta del modelo de gremios (Feeley 2003). Un estado favorecido correspondería si la distribución de las especies entre los gremios es uniforme dentro de las comunidades (Feeley 2003, Gotelli \& Entsminger 2003). Es importante indicar que para la realización de estas dos últimas pruebas, se excluyeron los datos obtenidos con la aplicación de las técnicas de captura de cebo humano y trampa lumínica de Shannon, ya que al utilizar atrayentes se genera un sesgo hacia las especies flebotominas "antropofílicas" y/o fotofílicas (Chaves \& Añez 2004).

Como algoritmo de aleatorización para la simulación, se escogió el "filas fijas- columnas fijas" (SIM9: fixed-fixed) (modelos nulos), en el cual las sumas de las filas y las de las columnas se mantienen fijas para la simulación, siendo un algoritmo menos proclive a cometer Errores Tipo I y II (Gotelli 2000, Gotelli \& Entsminger 2003). Se generaron 5000 matrices aleatorizadas usando un algoritmo tipo swap, el cual comienza con las matrices originales observadas y permuta sucesivamente sus subelementos, creando de esta manera nuevas matrices aleatorizadas y barajeadas (Stone \& Roberts 1990, Gotelli \& Entsminger 2003). Las pruebas de los modelos nulos de los C-score se llevaron a cabo utilizando el programa (software) de simulación ECOSIM versión 7.0 (Gotelli \& Entsminger 2004).

\section{Resultados}

Los análisis de simulación de los patrones de coocurrencia de las comunidades flebotominas realizados para diez poblaciones ubicadas en la zona bioclimática del BMST, y la localidad de "San Francisco" (escala reducida o local: domicilio, peridomicilio y área silvestre), revelaron que no existen resultados estadísticamente significativos ( $p>0.05$ ) entre las diferencias del $C$-score, su variación y los estados favorecidos y las medias del $C$-score, su variación y de los estados favorecidos del proceso de simulación; datos estos que se muestran en las Tablas 3, 4, 5 y 6.

\section{Discusión}

A una escala más amplia o regional, Chaves y Añez (2004) detectaron patrones de coocurrencia no agregados en comunidades flebotominas del estado Mérida, en la región andino-venezolana, al aplicar de igual modo modelos nulos, indicando que esto podría deberse a varios factores: competencia interespecífica, 
Tabla 3. Valores del índice $C$-score para las comunidades flebotominas a diferentes sitios de captura en 10 localidades endémicas ubicadas en el Bosque Muy Seco Tropical (BMST) del estado Falcón, Venezuela.

\begin{tabular}{lcc}
\hline Datos & C-Score & $\begin{array}{c}\text { Media } \pm \text { Varianza del } \\
\text { Índice C-Score simulado }\end{array}$ \\
\hline Sitios de captura, incluyendo todas las técnicas de captura & 0.10714 & $0.10714 \pm 0.00000$ \\
Sitios de captura, aspiración directa & 0.26667 & $0.26667 \pm 0.00000$ \\
Sitios de captura, papel aceitado & 0.06667 & $0.06667 \pm 0.00000$ \\
\hline
\end{tabular}

Tabla 4. Varianza del índice $C$-score y los valores del estado favorecido para las comunidades flebotominas con una estructura de gremio en especies antropofílicas $(A)$ y zoofílicas $(Z)$, a diferentes sitios de captura en 10 localidades endémicas ubicadas en el Bosque Muy Seco Tropical (BMST) del estado Falcón, Venezuela.

\begin{tabular}{|c|c|c|c|c|c|c|}
\hline Datos & $\begin{array}{l}\text { Varianza } \\
\text { del } \\
\text { C-Score }\end{array}$ & $\begin{array}{l}\text { Media } \pm \text { Varianza } \\
\text { simulada del } \\
\text { C-Score simulado }\end{array}$ & $\mathbf{P}$ & $\begin{array}{c}\text { Estados } \\
\text { favorecidos }\end{array}$ & $\begin{array}{c}\text { Media } \pm \text { Varianza de } \\
\text { los estados favorecidos } \\
\text { simulados }\end{array}$ & $\mathbf{P}$ \\
\hline $\begin{array}{l}\text { Sitios de captura, incluyendo } \\
\text { todas las técnicas de captura }\end{array}$ & 0.01389 & $0.03046 \pm 0.00081$ & 0.88900 & 1.00000 & $1.44600 \pm 0.43352$ & 0.90700 \\
\hline $\begin{array}{l}\text { Sitios de captura, aspiración } \\
\text { directa }\end{array}$ & 0.22222 & $0.10560 \pm 0.01519$ & 0.88900 & 1.00000 & $1.06000 \pm 0.51892$ & 0.76900 \\
\hline
\end{tabular}

Tabla 5. Valores del índice C-score para las comunidades flebotominas a diferentes sitios de captura en "San Francisco", estado Falcón, Venezuela.

\begin{tabular}{lccc}
\multicolumn{1}{c}{ Datos } & C-Score & Media \pm Varianza del Índice C-Score simulado & P \\
\hline $\begin{array}{l}\text { Sitios de captura, incluyendo todas } \\
\text { las técnicas de captura }\end{array}$ & 0.33333 & $0.38508 \pm 0.00290$ & 0.46360 \\
$\begin{array}{l}\text { Sitios de captura, aspiración directa } \\
\text { Sitios de captura, papel aceitado }\end{array}$ & 0.33333 & $0.33333 \pm 0.00000$ & 1.00000 \\
\hline
\end{tabular}

Tabla 6. Varianza del índice $C$-score y los valores del estado favorecido para las comunidades flebotominas con una estructura de gremio en especies antropofílicas (A) y zoofílicas (Z), a diferentes sitios de captura en "San Francisco", estado Falcón, Venezuela.

\begin{tabular}{cccccc}
\hline Datos & $\begin{array}{c}\text { Varianza } \\
\text { del } \text {-Score }\end{array}$ & $\begin{array}{c}\text { Media } \pm \text { Varianza } \\
\text { simulada del } \text {-Score } \\
\text { simulado }\end{array}$ & $\mathbf{P}$ & $\begin{array}{c}\text { Estados } \\
\text { favorecidos }\end{array}$ & $\begin{array}{c}\text { Media } \pm \text { Varianza de } \\
\text { lostados favorecidos } \\
\text { simulados }\end{array}$ \\
\hline $\begin{array}{c}\text { Sitios de captura, incluyendo } \\
\text { todas las técnicas de captura } \\
\text { Sitios de captura, aspiración } \\
\text { directa }\end{array}$ & 0.0000 & $0.18828 \pm 0.04498$ & 1.0000 & 0.0000 & $0.00000 \pm 1.24202$ \\
\hline
\end{tabular}

diversidad de requerimientos ambientales o por los procesos históricos de especiación particulares (Stone \& Roberts 1990). Por su parte, Chaves (2011) detectó a escala local que las comunidades flebotominas pueden cambiar de patrones de coocurrencia segregados en un bosque primario no intervenido, a uno agregado en una granja, sugiriendo que esto pudiera explicarse por el ajuste ecológico (ecological fitting) de los flebotominos en la selección de sitios de reposo diurno en ambientes intervenidos (Janzen 1985, Chaves 2011). Contrastando con estos hallazgos, cuando se hace el análisis de los patrones de coocurrencia a nivel local en el estado de Falcón, se revela que las comunidades flebotominas en los ambientes naturales y sinantrópicos (peri e intradomicilio) a nivel del BMST y de la población de "San Francisco" se encuentran agregadas, o sea existe mayor coocurrencia, tal como lo sugiere el hallazgo de que las diferencias entre los $C$-score calculados y los simulados no son estadísticamente significativas. En un intento por tratar de explicar este patrón de agregación (coocurrencia positiva), debemos indicar en primer lugar que las leishmaniasis no deberían considerarse en los actuales momentos como una parasitosis que el humano adquiere exclusivamente al adentrarse a sus ciclos enzooticos naturales, o ser considerada como una dolencia ocupacional relacionada con actividades profesionales en áreas zoonóticas; las evidencias actuales parecieran apuntar hacia cambios desde un punto de vista multidimensional, en los patrones geográficos, ecológicos, climáticos, socio-económicos y epidemiológicos en los patrones de trasmisión (Desjeux 2001, Chaves et al. 2008a, Miranda et al. 2009). La creciente urbanización producto de la descontrolada migración humana hacia la periferia de las ciudades, hace que la diferencia entre el peridomicilio y los ambientes naturales desaparezcan o sean más tenues: los flujos migratorios han pasado a ser en gran parte del medio urbano a medio urbano, 
y cada vez menos un flujo medio rural-medio rural o medio rural-medio urbano (Campbell-Lendrum et al. 2001, Desjeux 2001; Bejarano et al. 2001, 2002). Por lo tanto, el medio natural y los ambientes sinantrópicos pueden considerarse un espacio continuo, constituyéndose probablemente en ambientes más homogéneos, disminuyendo de este modo la segregación espacial y temporal (i.e, coocurrencia negativa) que puede ocasionar la heterogeneidad ambiental (Rosenzweig 1995). Estos cambios hacen que la conducta y composición de las comunidades de los transmisores y de los reservorios animales también se modifiquen, y éstos invadan a los nuevos ambientes sinantrópicos, sin la necesidad de una adaptación extrema, especialmente de las especies que poseen un amplio ajuste ecológico (Janzen 1985, Chaves 2011). Así, existen vectores o especies flebotominas con una alta fotofilia y que además pueden reposar en una gran variedad de sitios, que al ser atraídos a los ambientes humanos hacen más probable un mayor contacto flebotomino-humanoreservorios domésticos (Bejarano et al. 2001, 2002, Dos Santos et al. 2003, Chaves 2011). En este mismo orden de ideas, los reservorios silvestres, como por ejemplo Didelphis marsupialis (Marsupialia), poseen una amplia plasticidad en su adaptación a la vivienda humana donde merodean para alimentarse, convirtiéndose en un excelente reservorio domiciliario animal de Leishmania (Travi et al. 1994). Así mismo, deben evaluarse los animales domésticos, incluyendo aquellos que pueden ser reservorios domésticos del protozoo (e.g. perros) y otros vertebrados no susceptibles a Leishmania como las aves de corral, y que sirven de fuente alimentaria atrayente para las hembras flebotominas (Desjeux 2001, Alexander et al. 2002).

Por otra parte, la consideración del humano como hospedador incidental (i.e. vertedero) o posible reservorio (i.e. fuente y vertedero) intradomiciliar de leishmaniasis, es un factor a considerar en los nuevos esquemas eco-epidemiológicos de transmisión de las leishmaniasis (Chaves et al. 2008b). Estos nuevos cambios en los patrones epidemiológicos de las leishmaniasis en el continente americano ha llevado a Rotureau (2006) a preguntarse hipotéticamente en el título de su artículo: "Are New World leishmaniases becoming anthroponoses?”, tal como ha ocurrido esporádicamente en casos focalizados de la región paleotrópica (Reyburn et al. 2003). Lo discutido anteriormente, apoyaría la tesis según la cual las medidas para el control de las leishmaniasis deben implementarse o enfocarse desde un punto de vista de evitar el contacto con las hembras flebotominas, en vez de la intervención de las áreas silvestres (Chaves et al. 2008a, Chaves 2011).

En cuanto a la estructura de gremios (especies "antropofílicas" y "zoofílicas"), se encontró que la variación de $C$-score calculada no es significativamente diferente a la del modelo nulo simulado, lo que sugiere que todas las especies pertenecen a un mismo gremio. Similares hallazgos a los del presente estudio encontraron Chaves y Añez (2004) en la región andino-venezolana, aunque a escala regional. Es un hecho tangible en las áreas endémicas de leishmaniasis del Neotrópico, observar la coexistencia temporal y espacial, de especies flebotominas que pican al humano como otras que no, además de muchas clases de hospedadores vertebrados (Feliciangeli 1987, Young y Duncan 1994), pudiendo las hembras flebotominas adoptar una conducta de alimentación versátil y variable de amplia plasticidad, que depende de la disponibilidad de animales vertebrados (Bejarano et al. 2001, Travi et al. 2002).
Por ello, se puede indicar que la "preferencia" de una hembra flebotomina hacia un determinado hospedador (e.g. antropofilia), pudiera interpretarse como un evento heterogéneo, circunstancial y oportunista, donde las densidades de las hembras flebotominas (e.g. variaciones estacionales) y de los hospedadores vertebrados (movimientos) juegan un papel más fundamental (Kelly \& Thompson 2000, Chaves \& Añez 2004). Por lo tanto, como bien lo establecen Chaves y Añez (2004), no pareciera tener sentido utilizar la simple dicotomía discriminatoria de "especies antropofílicas" y "especies zoofílicas".

Por su parte, el análisis de los estados favorecidos indicó que las especies flebotominas no tienen a nivel local una regla de ensamble de uniformidad para la sustitución de los gremios. Por lo tanto, los resultados obtenidos en las comunidades flebotominas en diez localidades del BMST y de "San Francisco" de la región falconiana no parecieran apoyar la hipótesis de Chaves y Añez (2004), acerca de la probable existencia a escala local de especies introductoras (intruders) y especies esparcidoras (spreaders), al considerar que posiblemente las especies "zoofílicas" que se alimentan de los reservorios silvestres introducen (especies introductoras) los parásitos de Leishmania hacia los ambientes sinantrópicos, encargándose las especies "antropofílicas" de esparcir (especies esparcidoras) al protozoo dentro de las poblaciones humanas.

A pesar de lo discutido, se necesita indagar de una manera global y con mayores detalles los aspectos de la historia natural, biología y ecología del ecosistema para explicar con mayor eficacia y aproximación los patrones detectados.

\section{Agradecimientos}

Fundacite-Falcón (Proyecto S197-012, Decanato de Investigación, UNEFM, Coro, estado Falcón, Venezuela, y Tecana American University, Miami, Florida.

\section{Literatura citada}

Alexander B., R. de Carvalho, H. McCallum, et al. 2002. Role of the domestic chicken (Gallus gallus) in the epidemiology of urban visceral leishmaniasis in Brazil. Emerging Infectious Diseases 8:1480-1485.

Añez N., A. Rojas, E. Vargas, et al. 2012. Estudio epidemiológico sobre leishmaniasis visceral en la región semiárida del occidente de Venezuela con especial referencia a la detección de infecciones inaparentes. Boletín de Malariología y Salud Ambiental. 52: 245-256.

Bejarano E., S. Uribe, W. Rojas, et al. 2001. Presence of Lutzomyia evansi, a vector of American visceral leishmaniasis, in an urban area of the Colombian Caribbean coast. Transactions of the Royal Society of Tropical Medicine and Hygiene 95: 27-28. doi:10.1016/S0035-9203(01)90320-7

Bejarano E., S. Uribe, W. Rojas, et al. 2002. Phlebotomine sand flies (Diptera: Psychodidae) associated with the appearance of urban Leishmaniasis in the city of Sincelejo, Colombia. Memórias do Instituto Oswaldo Cruz 97: 645-647. doi: http://dx.doi.org/10.1590/S0074-02762002000500010

Campbell-Lendrum D., J. Dujardin, E. Martinez, et al. 2001. Domestic and peridomestic transmission of American cutaneous leishmaniasis: changing epidemiological patterns present new control opportunities. Memórias do Instituto Oswaldo Cruz 96: 159-162. doi: http://dx.doi.org/10.1590/S007402762001000200004

Cazorla D. \& P. Morales. 2012. Fauna flebotomina del estado Falcón, Venezuela (Diptera: Psychodidae). Revista peruana de biologia 19: 075 - 080 .

Chase J. 2003. Community assembly: when should history matter? Oecologia. 136: 489 - 498. doi: 10.1007/s00442-0031311-7 
Chaves L. \& N. Añez. 2004. Species co-occurrence and feeding behavior in sand fly transmission of American cutaneous leishmaniasis in western Venezuela. Acta Tropica 92: 219-224. doi: http://dx.doi.org/10.1016/j.actatropica.2004.08.001

Chaves L., J. Cohen, M. Pascual, et al. 2008a. Social exclusion modifies climate and deforestation impacts on a vector borne disease. PLOS Neglected Tropical Diseases 2: e176. doi: 10.1371/ journal.pntd.0000176

Chaves L., M. Hernández \& S. Ramos. 2008b. Simulación de modelos matemáticos como herramienta para el estudio de los reservorios de la Leishmaniasis Cutánea Americana. Divulgaciones Matemáticas. 16: 125-154.

Chaves L. 2011. Phlebotomine sand fly species co-occurrence at the local scale: differences between agricultural and forested areas. Boletín de Malariología y Salud Ambiental 51:35-39.

De Lima H., R. Borges, J. Escobar, et al. 2010. Leishmaniasis cutánea americana en Venezuela: un análisis clínico epidemiológico a nivel nacional y por entidad federal, 1988-2007. Boletín de Malariología y Salud Ambiental 50: 283-300.

Desjeux P. 2001. The increase in risk factors for leishmaniasis worldwide. Transactions of the Royal Society of Tropical Medicine and Hygiene 95: 239-243. doi: http://dx.doi.org/10.1016/ S0035-9203(01)90223-8

Dos Santos T., M. de Mello Gaia, R. Brazil, et al. 2003. Attraction of sand flies (Diptera: Psychodidae) to light traps in rural areas of Minas Gerais State, Brazil. Journal of the American Mosquito Control Association 19: 74-78.

Ewel J., A. Madriz \& Jr J. Tosi. 1976. Zonas de Vida de Venezuela. Memoria explicativa sobre el mapa ecológico. $4^{a}$ Ed. Caracas, Venezuela: Editorial Sucre.

Feeley K. 2003. Analysis of avian communities in Lake Guri, Venezuela, using multiple assembly rule models. Oecologia. 137: 104 - 113. doi: $10.1007 /$ s00442-003-1321-5

Feliciangeli M. 1987. Ecology of sandflies (Diptera: Psychodidae) in a restricted focus of cutaneous leishmaniasis in nothern Venezuela. I. - Description of the study area, catching methods and species composition. Memórias do Instituto Oswaldo Cruz 82: 119 - 124. doi: http://dx.doi.org/10.1590/S007402761987000100019

Fox B. 1987. Species assembly and evolution of community structure. Evolutionary Ecology 1: 201 - 213. doi: 10.1007/ BF02067551

González-Salazar C., C. Stephens \& P. Marquet. 2013. Comparing the relative contributions of biotic and abiotic factors as mediators of species' distribution. Ecological Modeling. 248: 57-70. doi: http://dx.doi.org/10.1016/j.ecolmodel.2012.10.007

Gordon C. 2000. The coexistence of species. Revista Chilena de Historia Natural 73: 175-198.
Gotelli N. 2000. Null model analysis of species co-occurrence patterns. Ecology. 81: 2606-2621.

Gotelli N. \& G. Entsminger. 2003. Swap algorithms in null model analysis. Ecology. 84: 532-535. doi: http://dx.doi. org/10.1890/0012-9658(2000)081 [2606:NMAOSC]2. $0 . \mathrm{CO} ; 2$

Gotelli N. \& G. Entsminger. 2004. ECOSIM: Null models software for ecology, Version 7.0. Acquired Intelligence Inc. and Kesey-Bear, Jericho, VT 05465. <http://garyentsminger. com/ecosim.htm>. Acceso 11/01/2008.

Herrera L. 2010. Una revisión sobre reservorios de Trypanosoma (Schizotrypanum) cruzi (Chagas, 1909), agente etiológico de la Enfermedad de Chagas. Boletín de Malariología y Salud Ambiental. 50: 3-15.

Janzen D. 1985. On ecological fitting. Oikos. 45: 308-310.

Kelly D. \& C. Thompson 2000. Epidemiology and optimal foraging: modelling the ideal free distribution of insect vectors. Parasitology. 120: 319- 327 .

Miranda A., R. Carrasco, H. Paz, et al. 2009. Molecular Epidemiology of American Tegumentary Leishmaniasis in Panama. The American Journal of Tropical Medicine and Hygiene 81: 565-571. doi: 10.4269/ajtmh.2009.08-0265

Reyburn H., M. Rowland, M. Mohammmed, et al. 2003. The prolonged epidemic of anthroponotic cutaneous leishmaniasis in Kabul, Afghanistan: 'bringing down the neighbourhood'. Transactions of the Royal Society of Tropical Medicine and Hygiene 97: 170-176. doi: http://dx.doi.org/10.1016/ S0035-9203(03)90111-8

Rotureau B. 2006. Are New World leishmaniases becoming anthroponoses? Medical Hypotheses 67: 1235-1241.

Rosenzweig M. 1995. Species diversity in space and time. Cambridge, England: Cambridge University Press.

Stone L. \& A. Roberts. 1990. The checkerboard score and species distributions. Oecologia. 85: 74-79. doi: 10.1007/BF00317345

Travi B., C. Jaramillo, J. Montoya, et al. 1994. Didelphis marsupialis, an important reservoir of Trypanosoma (Schizotrypanum) cruzi and Leishmania (Leishmania) chagasi in Colombia. The American Journal of Tropical Medicine and Hygiene 50: $557-565$.

Travi B., G. Adler, M. Lozano, et al. 2002. Impact of habitat degradation on phlebotominae (Diptera: Psychodidae) of tropical dry forests in Northern Colombia. Journal of Medical Entomology 39: 451-456. doi: http://dx.doi.org/10.1603/00222585-39.3.451

Vilchis O. 2000. Modelos nulos en interacciones biológicas, una propuesta: depredación en Crirostoma riojai-zooplancton. Ciencia Ergo Sum. 7: 153 - 156.

Young D. \& M. Duncan. 1994. Guide to the identification and geographic distribution of Lutzomyia sand flies in Mexico, the West Indies, Central and South America (Diptera: Psychodidae). Memories of the American Entomological Institute, Number 54. Associated Publishers, Gainesville, Florida, Estados Unidos de América. 